\title{
Erratum to: A diverse rodent fauna from the middle Bartonian (Eocene) of Les Alleveys, Switzerland: snapshot of the early theridomyid radiation
}

Jerry J. Hooker · Marc Weidmann

Published online: 28 May 2010

(C) Swiss Geological Society 2010

Erratum to: Swiss J Geosci (2007) 100:469-493

DOI 10.1007/s00015-007-1241-1

The Fig. 4 caption is repeated in Fig. 5, p. 475. The correct caption for Fig. 5 has to be read:

Fig. 5 Scanning electron micrographs of gold-palladium-coated epoxy casts of cheek teeth of Theridomyidae in occlusal view. a-e 'Protadelomys'? sp. from Les
Alleveys 1986; f P. lugdunensis from Lissieu; g-h Remys sp. from les Alleveys 1986. a Left $\mathrm{DP}^{4}$ ?, MGL. 47490; b left $\mathrm{M}^{3}$, MGL.47433; c right $\mathrm{M}_{1 / 2}$ (reversed), MGL.47599; d right $\mathrm{M}_{1 / 2}$ (reversed), MGL.47557; e right $\mathrm{M}_{3}$ (reversed), MGL. 47624; $f$ paratype right $\mathrm{M}_{1-3}$ (reversed), Faculté des Sciences Lyon, FSL.2628; $\mathrm{g}$ right $\mathrm{M}^{1 / 2}$ (reversed), MGL.47472; h right $\mathrm{M}^{1 / 2}$ (reversed), MGL.47601. Scale bar $1 \mathrm{~mm}$

The online version of the original article can be found under doi:10.1007/s00015-007-1241-1.

J. J. Hooker $(\square)$

Department of Palaeontology, Natural History Museum,

Cromwell Road, London SW7 5BD, UK

e-mail: j.hooker@nhm.ac.uk

M. Weidmann

Sentier du Molard 3, 1805 Jongny, Switzerland 